\title{
Validación de un simulador de conducción de bajo coste para el diseño de carreteras convencionales
}

\author{
David Llopis-Castelló \\ Grupo de Investigación en Ingeniería de Carreteras (GIIC), Universtitat Politècnica de \\ València \\ Francisco Javier Camacho-Torregrosa, Ana María Pérez-Zuriaga, Alfredo García \\ GIIC, Universitat Politècnica de València \\ Javier Marín-Morales \\ LabHuman, Universitat Politècnica de València \\ Juan F. Dols \\ Instituto de Diseño y Fabricación, Universitat Politècnica de València
}

\section{RESUMEN}

La cantidad de estudios de seguridad vial basados en simuladores de conducción está en continuo crecimiento. En este sentido, la Universitat Politècnica de València (UPV) ha

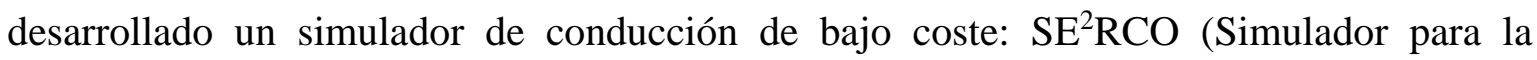
Evaluación, Entrenamiento y Rehabilitación de Conductores).

El principal objetivo de la investigación es la validación de este simulador, con el fin de desarrollar estudios relacionados con la seguridad vial y el diseño geométrico de carreteras incorporando el factor humano. Dicha validación se ha realizado a partir de la observación en campo de los perfiles continuos de velocidad desarrollados por 28 voluntarios conduciendo su propio vehículo en un tramo de carretera convencional de $30 \mathrm{~km}$. Los mismos voluntarios condujeron posteriormente en el simulador de conducción ese mismo tramo de carretera reconstruido en un entorno virtual.

Un total de 79 curvas y 52 rectas fueron objeto de análisis. La comparación entre las velocidades desarrolladas en la realidad y las observadas durante la simulación permitieron llevar a cabo la validez objetiva del simulador de conducción. Los resultados mostraron que la velocidad media en el simulador y en la realidad era similar cuando la velocidad simulada era inferior a $87.3 \mathrm{~km} / \mathrm{h}$. En caso de ser superior, la velocidad media en la realidad era menor que en el simulador. En cuanto a la velocidad de operación, se observó que la velocidad real era aproximadamente $5 \mathrm{~km} / \mathrm{h}$ menor que la simulada.

Finalmente, estos resultados estuvieron apoyados por la percepción de los conductores, ya que la mayoría de ellos evaluaron la calidad del entorno simulado y el grado de similitud entre la tarea de conducción real y simulada como medio o alto, consiguiendo de esta manera la validez subjetiva del simulador de conducción. 


\section{INTRODUCCIÓN}

En los últimos años, la preocupación por la seguridad vial ha crecido de manera significativa y comienza a reconocerse la necesidad de incorporar el factor humano para luchar con éxito contra la siniestralidad en carretera. El comportamiento de los conductores a lo largo de una carretera habitualmente es complejo y no intuitivo, pero no considerar su comportamiento en la fase de diseño puede provocar la pérdida de vidas humanas y un coste adicional de millones de euros en el caso de que se requiera una mejora después de ser construida.

Tres son los principales factores concurrentes en la siniestralidad: el factor humano, el vehículo y la infraestructura. Distintos estudios indican que el factor humano está presente en más del $90 \%$ de los accidentes ocurridos en carretera (Treat el al., 1979). Por tanto, es muy importante incluir dicho factor en el proceso de diseño de carreteras a pesar de que su análisis puede convertirse en una tarea difícil.

Actualmente, la mayoría de estudios relacionados con la seguridad vial y el diseño geométrico de carreteras emplean la velocidad de operación como medida sustitutiva del comportamiento del conductor. Esta velocidad es definida por la AASHTO (2011) como "la velocidad a la cual los conductores operan con sus vehículos en condiciones de flujo libre" y es llevada a la práctica comúnmente mediante el percentil 85 de la distribución de velocidad desarrollada por vehículos ligeros en condiciones de flujo libre y sin restricciones ambientales.

De esta forma, se pretende evaluar la seguridad vial de un tramo de carretera mediante la evaluación de la velocidad de operación desarrollada en el mismo. Esta velocidad puede observarse en tramos de carretera en fase de explotación, aunque su observación de forma continua puede conllevar altos costes. Sin embargo, durante la fase de planeamiento y diseño únicamente es posible estimarla en función de su geometría mediante modelos de velocidad de operación.

En este sentido, los simuladores de conducción pueden convertirse en una herramienta muy interesante y efectiva para incorporar el factor humano en la seguridad vial, pues son capaces de generar escenarios virtuales donde los conductores proporcionan una respuesta similar a la desarrollada en la carretera real. Estos escenarios pueden reproducir un tramo de carretera ya existente o un tramo en fase de diseño, permitiendo la observación de la velocidad de operación en ambos casos.

Además, en el caso de las carreteras en explotación, los simuladores de conducción presentan distintas ventajas frente a la toma de datos en campo: menores costes, menor riesgo y control de un mayor número de variables.

Por ello, ya son muchos los investigadores que han utilizado los simuladores de conducción para estudiar la influencia del diseño geométrico en el comportamiento del conductor. Estos 
estudios pueden ser agrupados en los siguientes ítems: coordinación del trazado en planta y en alzado (Bella, 2014; Easa et al., 2006; García et al., 2011); maniobra de adelantamiento (Toledo y Farah, 2011; Bella, 2011); carriles de aceleración y deceleración (Bella et al., 2007); estudio de la velocidad (Bella, 2008; Bella et al., 2014); diseño de intersecciones (Yan et al., 2008; Montella et al., 2010; Rossi et al., 2011); sección transversal (Bella, 2013; Yang et al., 2014; Rosey et al., 2009); evaluación de señalización (Daniels et al., 2010; Jamson y Lai, 2011).

Antes de ser utilizados en cualquier investigación relacionada con la seguridad vial o en la evaluación de la seguridad vial en un tramo de carretera, los simuladores de conducción deben ser validados con el fin de generar resultados válidos (Bella, 2009; Kaptein et al., 1996). El proceso de validación, que consiste en determinar el grado de similitud entre el comportamiento del conductor en la realidad y en el simulador, habitualmente se divide en:

- Validación objetiva: consiste en comparar los datos recogidos en el simulador con los recogidos en campo.

- Validación subjetiva: consiste en evaluar la percepción de los conductores.

La mayoría de los estudios centrados en la validación de simuladores de conducción para su empleo en investigaciones relacionadas con la seguridad vial se han basado en la comparación de la velocidad de operación en campo y en simulador (Bella, 2008). Esta comparación se ha focalizado en velocidades registradas en puntos concretos (centro de curvas y rectas), a pesar de que los simuladores permiten el registro continuo de la velocidad, debido a la dificultad de medir este parámetro de forma continua en campo.

A diferencia de los estudios anteriores, la investigación que se presenta muestra el proceso de validación del simulador de conducción $\mathrm{SE}^{2} \mathrm{RCO}$, basado en la comparación de los perfiles continuos de velocidad registrados en el simulador y los perfiles continuos de velocidad tomados en campo a través de la metodología presentada por Pérez-Zuriaga et al. (2010).

\section{OBJETIVOS E HIPÓTESIS}

El principal objetivo de esta investigación es la validación del simulador de conducción $\mathrm{SE}^{2} \mathrm{RCO}$ de la Universitat Politècnica de València para desarrollar estudios relacionados con la seguridad vial y el diseño geométrico de carreteras incluyendo el factor humano. Esta validación permitirá también su utilización en evaluaciones de la seguridad vial en tramos de carretera, tanto en fase de diseño como de explotación.

El proceso de validación considera dos dimensiones: validación objetiva y subjetiva. La primera de ellas se llevará a cabo a partir de la comparación entre las velocidades observadas en el simulador $\left(\mathrm{V}_{\mathrm{s}}\right)$ y las desarrolladas en campo $\left(\mathrm{V}_{\mathrm{r}}\right)$, mientras que la segunda se realizará considerando las percepciones de los conductores recogidas a partir de encuestas. 
La hipótesis fundamental del estudio es que existe una correlación entre $\mathrm{V}_{\mathrm{s}} \mathrm{y} \mathrm{V}_{\mathrm{r}}$, la cual permitirá la validación objetiva del simulador. Por otro lado, de acuerdo con Bittner et al. (2002) y Bella (2008), un aumento de $V_{s}$ se traduce en una mayor diferencia entre $V_{s}$ y $V_{r}$. Por último, según Bittner et al. (2002), se espera una menor $V_{s}$ en los elementos geométricos que presentan una alta carga de trabajo para los conductores.

\section{METODOLOGÍA}

La validación del simulador $\mathrm{SE}^{2} \mathrm{RCO}$ se basa en la comparación de los valores de diferentes parámetros relacionados con la velocidad de operación registrados durante la circulación de un grupo de conductores en un tramo de carretera y el valor de estos parámetros registrados durante la conducción de los mismos conductores en el simulador de conducción.

\subsection{Tramo de carretera de estudio}

El tramo de carretera seleccionado para el estudio discurre entre el PK 53+500 (Losa del Obispo) y el PK 83+700 (Titaguas) de la carretera CV-35, situada en la Comunidad Valenciana (España). Su longitud es aproximadamente de $30 \mathrm{~km}$ y presenta un ancho de carril de $3.25 \mathrm{~m}$, un ancho de arcén de $0.25 \mathrm{~m}$ y un volumen de tráfico de $2012 \mathrm{vh} /$ día.

Dicho tramo está compuesto por tres segmentos homogéneos claramente diferentes, cubriendo de esta forma un amplio rango de características geométricas (Tabla 1). Como resultado, fueron seleccionadas un total de 79 curvas horizontales aisladas y 52 rectas para el estudio.

\begin{tabular}{llllllll}
\hline Tramo homogéneo & $\mathbf{R}_{\min }$ & $\mathbf{R}_{\max }$ & $\mathbf{A R}$ & $\mathbf{A G}$ & $\mathbf{G}_{\max }$ & $\mathbf{L}_{\max }$ & $\mathbf{A L}$ \\
\hline Losa del Obispo-Chelva & 73 & 603 & 260 & $\pm 2.13 \%$ & $\pm 6.70 \%$ & 664 & 175 \\
Chelva-Tuéjar & 56 & 840 & 250 & $\pm 3.54 \%$ & $\pm 7.81 \%$ & 358 & 56 \\
Tuéjar-Titaguas & 37 & 483 & 145 & $\pm 4.33 \%$ & $\pm 10.28 \%$ & 1511 & 150 \\
\hline $\mathrm{R}_{\min }=$ radio mínimo (m); & $\mathrm{R}_{\max }=$ radio máximo (m); $\mathrm{AR}=$ radio medio $(\mathrm{m}) ; \mathrm{AG}=$ pendiente \\
media; $\mathrm{G}_{\max }=$ pendiente & máxima; & $\mathrm{L}_{\max }=$ longitud máxima de rectas (m); $\mathrm{AL}=$ longitud \\
media de rectas (m)
\end{tabular}

Tabla 1 - Características geométricas del tramo de estudio

\subsection{Voluntarios}

En el estudio participaron de forma voluntaria 28 conductores, estando su edad comprendida entre 21 y 61 años, con una distribución entre hombres y mujeres de 80/20.

Las distribuciones de edad y género de los participantes fueron representativas de los conductores reales de esa región, puesto que eran similares a las distribuciones observadas por Pérez-Zuriaga et al. (2010). En este estudio anterior se obtuvieron las distribuciones de edad y género de más de 80 usuarios (no voluntarios) del mismo tramo de carretera. Además, García et al. (2015) comparó los perfiles de velocidad desarrollados por los voluntarios del presente estudio con los de los conductores reales observados en el mencionado estudio anterior, validando de esta forma el uso de los datos de los participantes. 


\subsection{Estudio de campo}

\subsubsection{Toma de datos}

La toma de datos en campo fue desarrollada por García et al. (2015) a partir de una metodología quasi-naturalística. Cada uno de los voluntarios condujo su propio vehículo por el tramo de estudio, tanto en sentido de ida como de vuelta.

Cada uno de los vehículos se equipó con 3 cámaras VIRB Elite, que tienen incorporado una unidad GPS. Estas cámaras grababan la carretera en HD y recogían datos de posición (1 Hz), velocidad y aceleración $(10 \mathrm{~Hz})$.

A partir de los datos de posición recogidos fueron desarrollados los perfiles de velocidad de operación de cada uno de los conductores. Además, la experiencia en la conducción, la familiaridad con el tramo de carretera, el mareo y la carga de trabajo fueron cuestiones que se preguntaron a todos los participantes por medio de una encuesta.

\subsubsection{Reducción de datos}

Una vez obtenidos los datos, el primer paso fue transformarlos de referencia temporal a espacial a partir de un programa desarrollado por el grupo de investigación. Además, el trazado en planta del tramo de carretera de estudio fue restituido a partir del procedimiento propuesto por Camacho-Torregrosa et al. (2015).

Por otra parte, los datos relativos a tramos en los que los conductores no habían operado en condiciones de flujo libre fueron eliminados. Para ello se empleó la metodología propuesta por Pérez-Zuriaga et al. (2013), que se basa en la hipótesis de que cuando un conductor experimenta una variación brusca y repentina respecto a la tendencia del percentil de velocidad de operación más cercano a su comportamiento es debido a que no opera en condiciones de flujo libre. Después de este paso, el $66 \%$ de los datos recogidos en curvas y el $68 \%$ de los recogidos en rectas fueron válidos.

\subsection{Estudio en el simulador de conducción}

\subsubsection{Simulador de conducción $\mathrm{SE}^{2} \mathrm{RCO}$}

$\mathrm{SE}^{2} \mathrm{RCO}$ es un simulador de conducción de base fija (Figura 1). Este está compuesto por un ordenador de simulación; recogida de datos en tiempo real; router inalámbrico; 3 pantallas de $1.80 \times 0.34 \mathrm{~m}$ con $120^{\circ}$ de campo de visión; sistema de sonido estéreo; volante, pedales y cambio de marchas de un Citroen Saxo; y asiento genérico ajustable.

Todo ello hace que el simulador ofrezca unas condiciones de conducción muy similares a las reales, siendo el escenario virtual muy realista. Además, el simulador permite recoger variables como la velocidad longitudinal y lateral, la localización y la dirección con una frecuencia de $10 \mathrm{~Hz}$. Después de la toma de datos, todas las variables fueron transformadas de referencia temporal a espacial usando el programa desarrollado por el grupo de 
investigación.
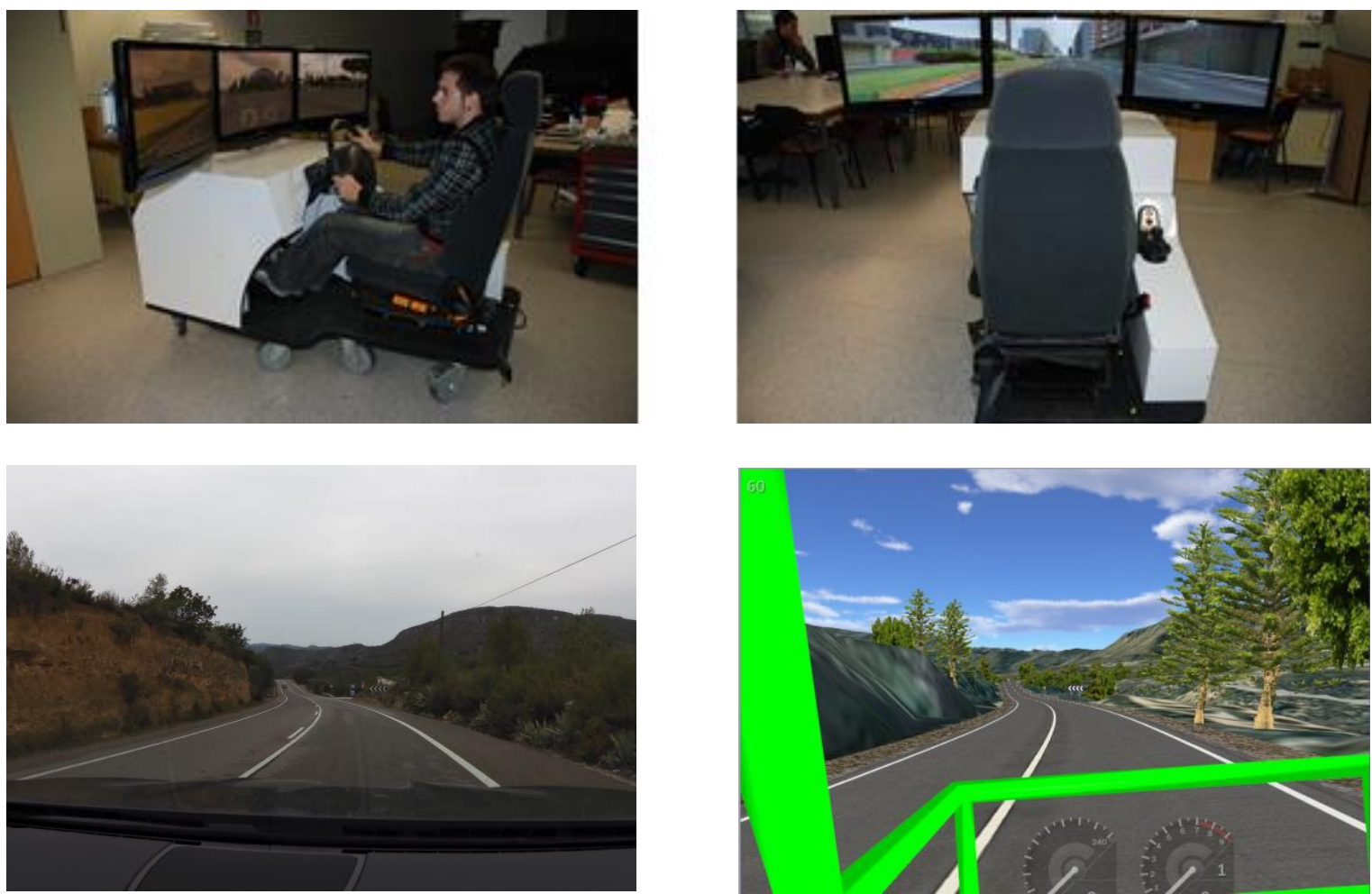

Fig. 1 - Simulador de conducción $\mathrm{SE}^{2} \mathrm{RCO}$

\subsubsection{Desarrollo del entorno virtual}

Previamente a la realización de las pruebas en el simulador de conducción fue necesario construir el entorno virtual, el cual fue desarrollado a partir de:

- Trazado en planta y alzado del tramo de carretera de estudio

- Modelo de superficie e imagen aérea del entorno

- Inventario de los elementos de la carretera y su entorno

Tanto el trazado en planta como en alzado fue obtenido a partir de la metodología propuesta por Camacho-Torregrosa et al. (2015). Por otro lado, los diferentes elementos de la carretera y su entorno, tales como árboles, señalización o barreras de seguridad, fueron localizados en un archivo CAD e implementados en el simulador. De esta forma el simulador ofreció un escenario virtual muy cercano a las condiciones reales de la carretera y su entorno.

\subsubsection{Toma de datos}

El objetivo de la toma de datos en el simulador de conducción era el mismo que en campo, pero la metodología fue distinta. En este caso se siguieron los siguientes pasos:

1. Información: los conductores eran informados acerca de cómo debían realizar la prueba.

2. Encuesta previa: los voluntarios debían rellenar una encuesta acerca de su experiencia como conductores.

3. Entrenamiento: la sesión de entrenamiento era necesaria para asegurar que los 
conductores se adaptaban correctamente a los controles del simulador y su entorno. Esta sesión consistía en conducir por un tramo de carretera similar al tramo de estudio durante al menos $10 \mathrm{~min}$.

4. Toma de datos: conducción de un trayecto de ida y de vuelta por el tramo de carretera de estudio.

5. Encuesta final: los participantes debían rellenar una segunda encuesta acerca de los síntomas de adaptación a la simulación y la percepción del entorno (Tabla 2).

\begin{tabular}{|c|c|}
\hline Síntomas de Adaptación a la Simulación & Percepciones de los conductores \\
\hline Mareo & Realidad del entorno simulado \\
\hline Náuseas & $\begin{array}{c}\text { Similitud las tareas de conducción en el } \\
\text { simulador y la realidad }\end{array}$ \\
\hline Cansancio ocular & Naturalidad en la conducción \\
\hline Dolor de cabeza & Carga de trabajo \\
\hline Somnolencia & Familiaridad con el tramo de carretera \\
\hline
\end{tabular}

Tabla 2 - Encuesta final

Con el fin de prevenir mareos, se instó a todos los participantes a levantarse y caminar durante varios minutos entre la sesión de entrenamiento y la prueba, así como entre el trayecto de ida y el de vuelta. En este sentido, los investigadores anotaban si el conductor mostraba algún síntoma de mareo a lo largo de la prueba.

Por último, señalar que la prueba en el simulador de conducción fue realizada por 24 de los 28 voluntarios que participaron en la prueba de campo. Asimismo, 3 de estos participantes presentaron problemas relacionados con mareos y náuseas, por lo que finalmente se procesaron los datos obtenidos de 21 conductores.

\section{ANÁLISIS}

Una vez realizada la toma de datos en campo y en el simulador de conducción, se llevaron a cabo dos análisis diferenciados. Por un lado, se realizó una comparación entre las velocidades observadas en campo y en el simulador a través de un análisis exploratorio previo y un análisis estadístico posterior. Por otro lado, también fueron analizadas las percepciones de los conductores a partir de los resultados obtenidos en las encuestas realizadas en ambas tomas de datos.

\subsection{Velocidad}

\subsubsection{Análisis exploratorio}

El primer paso fue comparar las distribuciones de velocidad de operación obtenidas en el entorno real y simulado. Para ello se analizaron los percentiles 15, 30, 50, 70 y 85 de la velocidad simulada $\left(\mathrm{V}_{\mathrm{s}}\right)$ y la velocidad real $\left(\mathrm{V}_{\mathrm{r}}\right)$. La figura 2 muestra que estos percentiles son muy similares en aquellos elementos de la carretera que actúan de control geométrico para los conductores, es decir, la diferencia entre $V_{s}$ y $V_{r}$ es menor en curvas que en rectas. 
Esta conclusión es similar a la obtenida por Bella (2008) y Bittner et al. (2002), quienes indicaron que existe una mayor variabilidad de la velocidad en el simulador de conducción.

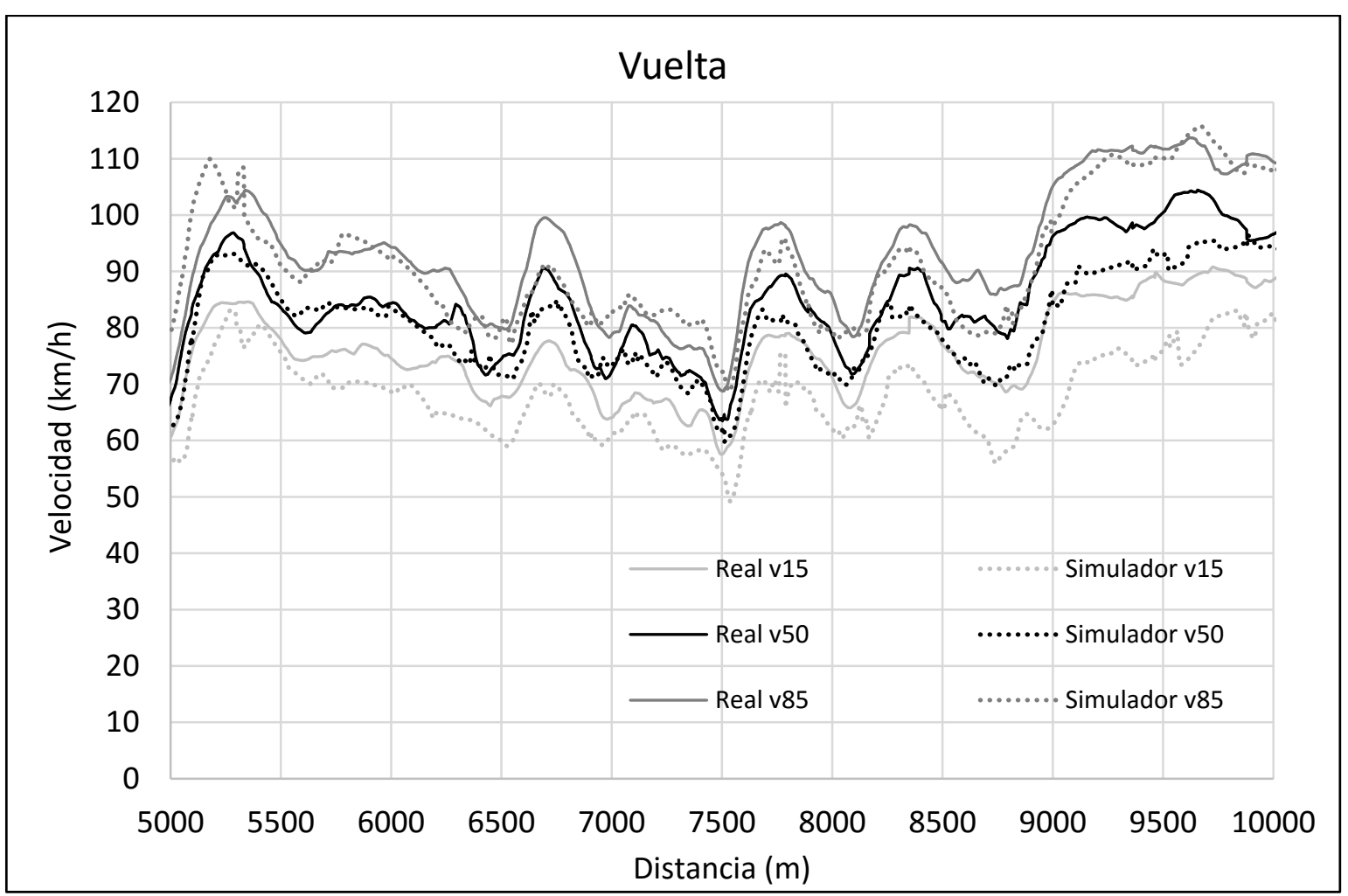

Fig. 2 - Comparación entre $V_{s}$ y $V_{r}$

Asimismo, también fueron analizadas las velocidades medias y de operación en cada una de las curvas y rectas. Para ello, la velocidad seleccionada en cada curva era la mínima observada, mientras que en el caso de las rectas se seleccionó la máxima observada. La identificación de estas velocidades fue posible gracias a la disponibilidad de los perfiles continuos de velocidad tanto en el entorno real como simulado.

Las curvas horizontales imponen un control geométrico para los conductores, por lo que la velocidad mínima permite determinar cuánto control geométrico presenta cada una de ellas. Por otro lado, las rectas no presentan ningún control geométrico sobre los conductores, por lo que los conductores pueden experimentar mayores velocidades. Por ello, en rectas se seleccionaron las velocidades máximas.

Una vez identificadas estas velocidades, se analizó la diferencia entre las velocidades observadas en el simulador y en la carretera real $\left(\mathrm{V}_{\mathrm{s}}-\mathrm{V}_{\mathrm{r}}\right)$. En curvas, la diferencia entre las velocidades medias $\left(\mathrm{V}_{\mathrm{ms}}-\mathrm{V}_{\mathrm{mr}}\right)$ se situó entre $-9.92 \mathrm{~km} / \mathrm{h}$ y $+8.65 \mathrm{~km} / \mathrm{h}$, mientras que la diferencia entre las velocidades de operación ( $\mathrm{V}_{85 \mathrm{~s}}-\mathrm{V}_{85 \mathrm{r}}$ ) osciló entre $-7.12 \mathrm{~km} / \mathrm{h}$ y +15.70 $\mathrm{km} / \mathrm{h}$. Con respecto a las rectas, $\left(\mathrm{V}_{\mathrm{ms}}-\mathrm{V}_{\mathrm{mr}}\right)$ se situó entre $-7.12 \mathrm{~km} / \mathrm{h} \mathrm{y}+16.14 \mathrm{~km} / \mathrm{h}$, mientras que $\left(\mathrm{V}_{85 \mathrm{~s}^{-}} \mathrm{V}_{85 \mathrm{r}}\right)$ presentó valores entre $-8.76 \mathrm{~km} / \mathrm{h}$ y $+27.84 \mathrm{~km} / \mathrm{h}$. 


\subsubsection{Análisis estadístico}

Para determinar si las velocidades observadas en el simulador pueden considerarse similares a las observadas en campo se llevó a cabo un test de hipótesis. Para cada elemento, se formularon las siguientes hipótesis: (a) Hipótesis nula $\mathrm{H}_{0}: \mathrm{V}_{\mathrm{ms}}=\mathrm{V}_{\mathrm{mr}}$; (b) Hipótesis alternativa $\mathrm{H}_{1}: \mathrm{V}_{\mathrm{ms}} \neq \mathrm{V}_{\mathrm{mr}}$. El nivel de confianza considerado en el análisis fue del $95 \%$.

Destacar que la hipótesis nula fue aceptada en más del $90 \%$ de los elementos estudiados. Solo en 7 curvas y 6 rectas dicha hipótesis fue rechazada, principalmente debido a que la velocidad observada en estos elementos estaba influenciada por factores externos, tales como la proximidad de intersecciones importantes o algunos errores puntuales relacionados con el escenario simulado.

Así pues, se identificó una correlación entre las velocidades observadas en campo y en el simulador, lo que permitió la validación objetiva del simulador de conducción.

\subsection{Percepción de los conductores}

Para estudiar la percepción de los conductores en el simulador de conducción se tuvieron en cuenta los datos tomados en las encuestas.

Los resultados mostraron que el $62.5 \%$ y el $33.33 \%$ de los conductores evaluaron la calidad del entorno virtual como medio y alto, respectivamente. Además, el $80 \%$ de los voluntarios indicaron que el grado de similitud entre la tarea de conducción real y en el simulador era media o alta. Finalmente, cabe destacar que la mayoría de participantes evaluaron la carga de trabajo y la naturalidad en la conducción como media, siendo estos resultados similares a los obtenidos en el estudio de campo.

Por tanto, se logró también la validación subjetiva del simulador de conducción, puesto que este fue capaz de generar un entorno y una conducción prácticamente similar a la experimentada en la carretera real.

\section{DISCUSIÓN}

Para concluir la validación, se analizaron las velocidades medias y de operación en el entorno real y simulado para curvas y rectas, tanto separada como conjuntamente.

Con respecto a las curvas, se observó una estrecha relación entre las velocidades observadas en campo y en el simulador, siendo la velocidad de operación en el simulador mayor que la observada en la carretera real (Figura 3b). Este hecho también fue observado por la mayor parte de estudios de validación previos, y muy posiblemente se deba a la falta de aceleración lateral y de riesgo generada por el simulador de conducción (Bella, 2008).

Por otra parte, las velocidades medias fueron similares, siendo este un resultado destacable (Figura 3a). Este hecho podría explicarse teniendo en cuenta cómo opera un conductor 
medio. Bajo estas condiciones, los conductores experimentan una aceleración lateral inferior a la que experimenta un conductor operando en el percentil 85 de velocidad, por lo que la diferencia con respecto al entorno simulado es inferior o casi inexistente.
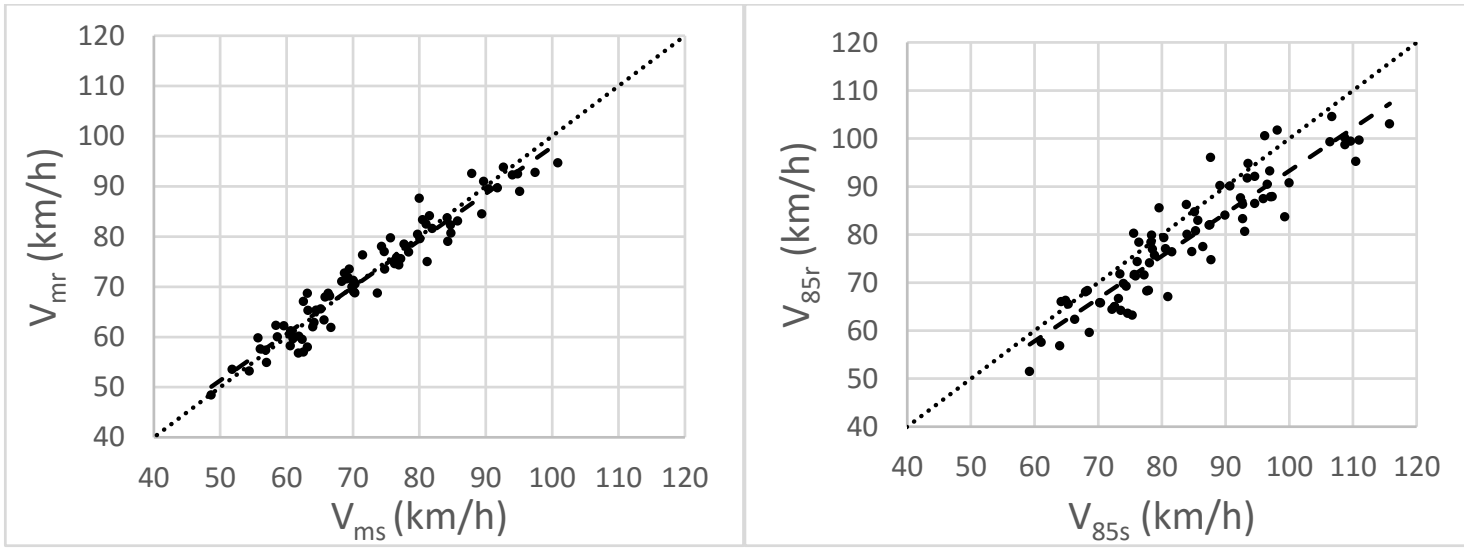

Fig. 3 - Velocidad media y de operación en curvas

En cuanto a rectas, las relaciones observadas entre ambas velocidades son mucho más débiles que las observadas en curvas, principalmente debido a la falta de riesgo experimentada en el simulador de conducción (Figura 4). Así, tanto la velocidad media como la velocidad de operación observada en el simulador fue superior a las observadas en campo.
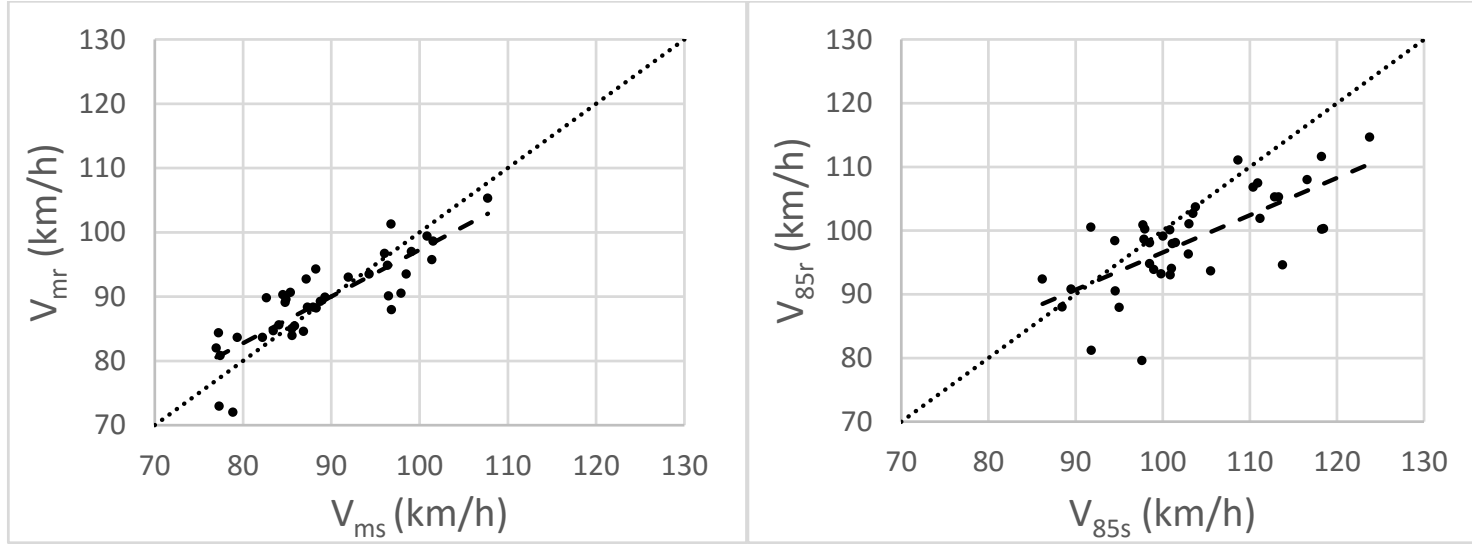

Fig. 4 - Velocidad media y de operación en rectas

Considerando las velocidades en curvas y rectas conjuntamente, se identificó que a medida que la velocidad era mayor, también lo era la diferencia entre la velocidad real y la simulada (Figura 5). En otras palabras, a medida que el trazado era más complejo, menores eran las diferencias entre ambas velocidades. Dichos resultados eran consistentes con las investigaciones llevadas a cabo por Bella (2008) y Bittner et al. (2002).

A pesar de que el análisis estadístico de la velocidad indicó que las velocidades medias eran similares, se observó un ligero cambio de tendencia cuando la velocidad simulada era mayor de $90 \mathrm{~km} / \mathrm{h}$ aproximadamente. Debido a ello, se propuso un modelo lineal compuesto, en el que para velocidades simuladas inferiores a $87.66 \mathrm{~km} / \mathrm{h}$ las velocidades medias eran 
similares, pero para mayores velocidades la velocidad real era inferior que la simulada (Figura 5a).

Asimismo, un segundo modelo fue calibrado para determinar la velocidad de operación real. En este sentido, dicha velocidad era $4.86 \mathrm{~km} / \mathrm{h}$ inferior que la velocidad simulada (Figura $5 b)$.
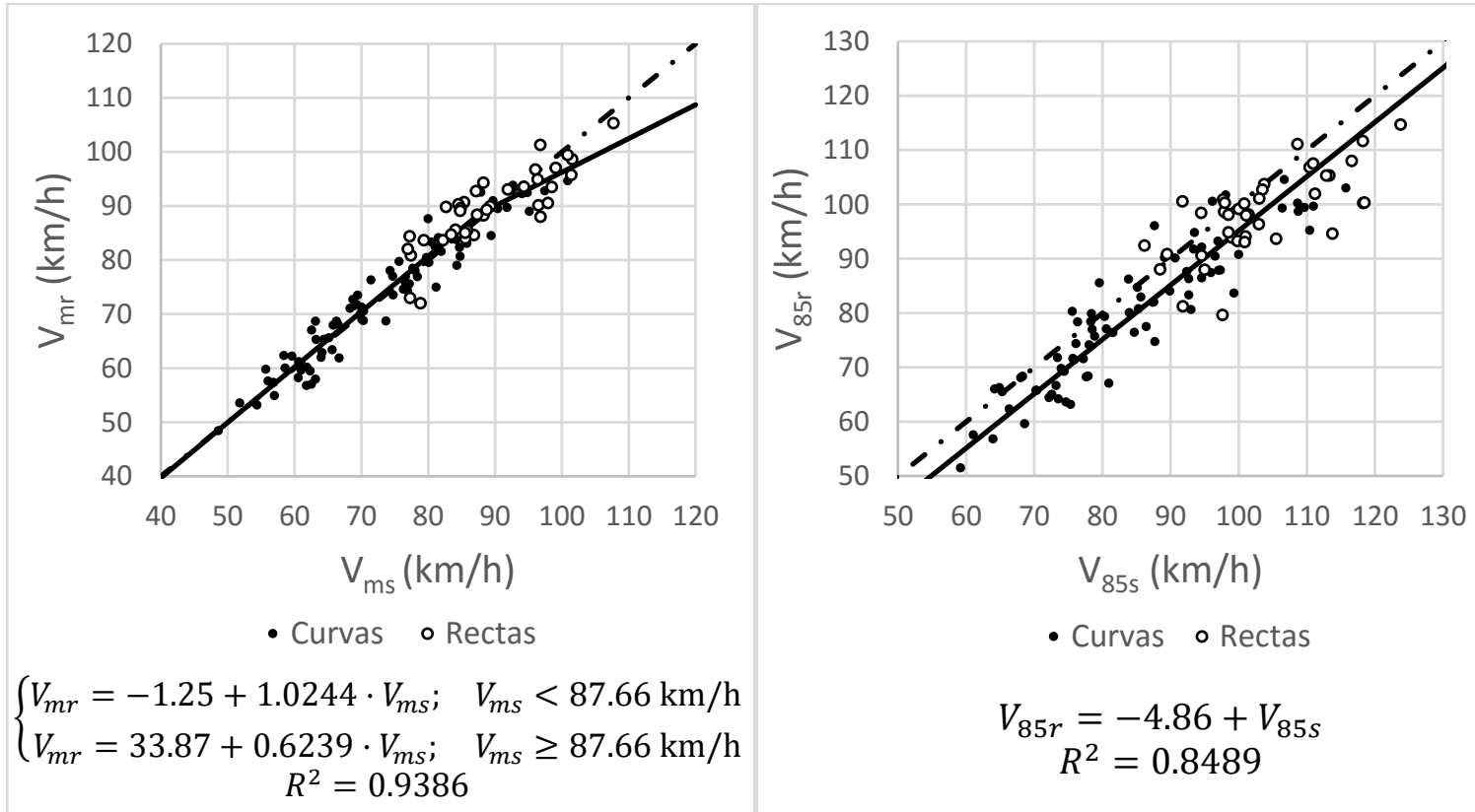

Fig. 5 - Modelos de velocidad media y de operación

Estos dos modelos de regresión permiten estimar las velocidades reales a partir de las velocidades observadas en el simulador. De esta forma, los ingenieros podrán estimar la velocidad real de carreteras en fase de diseño a partir de la velocidad desarrollada en el simulador.

\section{CONCLUSIONES}

La validación del simulador de conducción $\mathrm{SE}^{2} \mathrm{RCO}$ ha sido llevada a cabo a partir de la comparación de los perfiles de velocidad continuos y la percepción de los conductores obtenidos en campo y en el propio simulador de conducción.

Por un lado, fueron identificadas la velocidad media y de operación en 79 curvas con radios comprendidos entre 40 y $520 \mathrm{~m}$ y en 52 rectas con longitudes entre 120 y $1500 \mathrm{~m}$. A partir de la comparación de las velocidades obtenidas en campo y en el simulador de conducción se logró la validación objetiva del simulador. En este sentido, la velocidad de operación real era $4.86 \mathrm{~km} / \mathrm{h}$ inferior a la velocidad de operación simulada. Considerando las velocidades medias, estas eran similares cuando la velocidad simulada era inferior a $87.66 \mathrm{~km} / \mathrm{h}$, mientras que para valores superiores la velocidad media real era inferior a la simulada. 
Por otro lado, el análisis de las percepciones de los conductores en el entorno simulado y en el real permitió la validez subjetiva del simulador de conducción. De hecho, la gran parte de los voluntarios evaluaron la calidad del entorno simulado y el grado de similitud entre la tarea de conducción real y simulada como media o alta. Además, los resultados obtenidos con respecto a la carga de trabajo del conductor y la naturalidad en la conducción fueron similares a los observados en campo.

Así pues, el simulador $\mathrm{SE}^{2} \mathrm{RCO}$ es una herramienta útil para llevar a cabo investigaciones relacionadas con la seguridad vial y el diseño geométrico de carreteras que incluyan el factor humano, puesto que existe una alta correlación entre el comportamiento del conductor en el simulador y la carretera real. Del mismo modo, el simulador $\mathrm{SE}^{2} \mathrm{RCO}$ puede emplearse para la evaluación de la seguridad vial de un carretera desde la fase de diseño hasta la fase de explotación.

\section{AGRADECIMIENTOS}

Los autores quisieran agradecer a la Universitat Politècnica de València (UPV), que financió el proyecto de investigación "CONSIM - Desarrollo de un Modelo para la Evaluación de la Consistencia del Diseño Geométrico de Carreteras Convencionales mediante Simuladores de Conducción" (PAID 05-2012). Asimismo, agradecer también al Ministerio de Economía y Competitividad y al Fondo Social Europeo, que financiaron el proyecto de investigación "CASEFU - Estudio experimental de la funcionalidad y seguridad de las carreteras convencionales" (TRA2013-42578-P), del cual forma parte este estudio.

\section{REFERENCIAS}

AASHTO (2011). A Policy on Geometric Design of Highways and Streets. American Association of State Highway and Transportation Officials.

BELLA, F. (2008). Driving simulator for speed research on two-lane rural roads. Accident Analysis and Prevention 40(3), pp. 1078-1087.

BELLA, F. (2009). Can Driving Simulators Contribute to Solving Critical Issues in Geometric Design? Transportation Research Record: Journal of the Transportation Research Board 2138, pp. 120-126.

BELLA, F. (2011). How traffic conditions affect driver behavior in passing maneuver. Advances in Transportation Studies, pp. 113-126.

BELLA, F. (2013). Driver perception of roadside configurations on two-lane rural roads: Effects on speed and lateral placement. Accident Analysis and Prevention 50, pp. 251-262. BELLA, F. (2014). Driver perception hypothesis: Driving simulator study. Transportation Research Part F: Traffic Psychology and Behaviour 24, pp. 183-196.

BELLA, F., CALVI, A., y D'AMICO F. (2014). Analysis of driver speeds under night driving conditions using a driving simulator. Journal of Safety Research 49, pp. 45-52.

BELlA, F., GARCÍA, A., SOLVES, F., y ROMERO, M. A. (2007). Driving simulator validation for deceleration lane design. Transportation Research Board 86th Annual Meeting, 07-0894. 
BITTNER, A., SIMSEK, O., LEVISON, W., y CAMPBELL J. (2002). On-Road Versus Simulator Data in Driver Model Development Driver Performance Model Experience. Transportation Research Record: Journal of the Transportation Research Board 1803, pp. $38-44$.

CAMACHO-TORREGROSA, F. J., PÉREZ-ZURIAGA, A. M., CAMPOY-UNGRÍA, J. M., GARCÍA, A., y TARKO, A. P. (2015). Use of Heading Direction for Recreating the Horizontal Alignment of an Existing Road. Computer-Aided Civil and Infrastructure Engineering 30(4), pp. 282-299.

DANIELS, S., VANRIE, J., DREESEN, A., y BRIJS, T. (2010). Additional road markings as an indication of speed limits: Results of a field experiment and a driving simulator study. Accident Analysis and Prevention 42(3), pp. 953-960.

EASA, S. M., y HE, W. (2006). Modeling Driver Visual Demand on Three-Dimensional Highway Alignments. Journal of Transportation Engineering 132(5), pp. 357-365.

GARCÍA, A., CAMACHO-TORREGROSA, F. J., y MARÍN-MORALES, J. (2015). Experimental determination of the inertial operating speed for consistency and segmentation analysis. Transportation Research Board 94th Annual Meeting, 15-4079.

GARCÍA, A., TARKO, A., DOLS RUÍZ, J. F., MORENO CHOU, A. T., y CALATAYUD, D. (2011). Analysis of the influence of 3D coordination on the perception of horizontal curvature using driving simulator. Advances in Transportation Studies 24, pp. 33-44.

JAMSON, S., y LAI F. (2011). Are Novelty Effects of Road Safety Treatments Observable in Simulator Experiments? Transportation Research Board 90th Annual Meeting, 11-0535. KAPTEIN, N., THEEUWES, J. y VAN DER HORST, R. (1996). Driving Simulator Validity: Some Considerations. Transportation Research Record: Journal of the Transportation Research Board 1550, pp. 30-36.

MONTElla, A., ARIA, M., D’AMBrosio, A., GALANTE, F., MAURIELlO, F., y PERNETTI, M. (2010). Perceptual Measures to Influence Operating Speeds and Reduce Crashes at Rural Intersections. Transportation Research Record: Journal of the Transportation Research Board 2149, pp. 11-20.

PÉREZ-ZURIAGA, A. M., CAMACHO-TORREGROSA, F. J., GARCÍA, A., y CAMPOY-UNGRÍA, J. M. (2013). Application of global positioning system and questionnaires data for the study of driver behaviour on two-lane rural roads. IET Intelligent Transport Systems 7(2), pp. 182-189.

PÉREZ-ZURIAGA, A., GARCÍA, A., CAMACHO-TORREGROSA, F. J., y D’ATTOMA, P. (2010). Modeling Operating Speed and Deceleration on Two-Lane Rural Roads with Global Positioning System Data. Transportation Research Record: Journal of the Transportation Research Board 2171, pp. 11-20.

ROSEY, F., AUBERLET, J.M., MOISAN, O., y DUPRÉ, G. (2009). Impact of Narrower Lane Width: Comparison Between Fixed-Base Simulator and Real Data. Transportation Research Record: Journal of the Transportation Research Board 2138(1), pp. 112-119.

ROSSI, R., GASTALDI, M., MENEGUZZER, C., y GECCHELE, G. (2011). GapAcceptance Behavior at Priority Intersection: Field Observations Versus Experiments with Driving Simulator. Transportation Research Board 90th Annual Meeting, 11-3241. 
TOLEDO, T., y FARAH, H. (2011). Alternative Definitions of Passing Critical Gaps. Transportation Research Record: Journal of the Transportation Research Board 2260(1), pp. 76-82.

TREAT, J.R., TUMBAS, N.S., MCDONALD, S.T., SHINAR, D., HUME, R.D., MAYER, R.E., STANSIFER, R.L. y CASTELLAN, N.J.. Tri-level study of the causes of traffic accidents: Final report - Executive summary. Report DOT-5 HS-034-3-535-79-TAC(S). Bloommington. Institute for Research in Public Safety, 1979.

YAN, X., ABDEL-ATY, M., RADWAN, E., WANG, X., y CHILAKAPATI, P. (2008). Validating a driving simulator using surrogate safety measures. Accident Analysis and Prevention 40(1), pp. 274-288.

YANG, Q., OVERTON, R., y RICHARDS, S. H. (2014). Driver Behaviors on Rural Highways with and without Curbs - A Driving Simulator Based Study. International journal of injury control and safety promotion 21(2), pp. 115-126. 